Res Publica. Revista de Historia de las Ideas Políticas

ISSN: $1576-4184$

\title{
El buen vivir como alternativa a un modelo civilizador en crisis
}

\author{
Sara Ferreiro Lago*1
}

Recibido: 4 de junio de 2018 / Aceptado: 1 de noviembre de 2018

Resumen. La idea de Buen Vivir según los movimientos indigenistas de América Latina (Abya Yala) es una cosmovisión ancestral de la vida que tiene su origen en los saberes y prácticas tradicionales. Hace referencia a un vivir en armonía con los seres humanos y la naturaleza, desde una mirada no egocéntrica ni antropocéntrica, que reconocen nuestra interdependencia y ecodependencia. Esta noción resurge con fuerza en las últimas décadas como una alternativa al sistema capitalista, al mito del desarrollo $\mathrm{y}$ del crecimiento económico, jugando un papel importante en movimientos sociales y en iniciativas gubernamentales de varios Estados latinoamericanos.

Palabras clave: Crisis civilizatoria; Buen vivir; Abya Yala.

\section{[en] Good Living as an Alternative to a Civilizing Model in Crisis}

\begin{abstract}
The idea of Good Living according to the indigenous movements of Latin America (Abya Yala) is an ancestral worldview of life that has its origin in traditional knowledge and practices. It refers to living in harmony with human beings and nature, from a non-egocentric or anthropocentric view, which recognizes our interdependence and eco-dependence. This notion has re-emerged with force in the last decades as an alternative to the capitalist system, to the myth of development and economic growth, playing an important role in social movements and governmental initiatives of several Latin American States.
\end{abstract}

Keywords: Civilizational Crisis; Good Living; Abya Yala.

Sumario: 1. La crisis civilizatoria como una crisis multidimensional. 2. La búsqueda de alternativas a un modelo socioeconómico en crisis. 3. Concepciones del Buen Vivir en América Latina. 3.1. El Buen Vivir como alternativa al desarrollo. 3.2. Reconceptualizaciones del Buen Vivir por parte de los feminismos comunitarios en América Latina. 3.3. El Buen Vivir como horizonte de cambio a escala global. 4. El descentramiento de occidente como paradigma civilizador.

Cómo citar: Ferreiro Lago, S. (2018). El buen vivir como alternativa a un modelo civilizador en crisis, en Res publica 21.3, 559-569.

\footnotetext{
* Universidad Complutense de Madrid saraferreirolago@gmail.com

1 Personal investigador en formación en el Departamento de Lógica y Filosofía Teorética de la Universidad Complutense de Madrid.
} 


\section{La crisis civilizatoria como una crisis multidimensional}

La crisis civilizatoria es enunciada en nuestros días para referirse a una crisis a nivel de la estructura de la organización social y económica que afecta a múltiples dimensiones de la vida. Francisco Fernández Buey, en un texto escrito tras el estallido de la crisis financiera de 2008, la describe de la siguiente manera:

La noción de crisis de civilización se ha divulgado y popularizado en los últimos tiempos sin que a menudo se defina o explique. En la actualidad, es preciso matizar que se trata de la crisis de la civilización capitalista y que afecta al conjunto de conocimientos y costumbres que constituye lo que suele definirse como civilización. Los rasgos centrales de esta son la crisis ecológica, el hecho de que vivimos en un mundo globalizado y la homogeneización cultural bajo la forma de occidentalización del mundo. Nos encontramos ante un desastre ético por lo que los valores vigentes y establecidos en nuestras sociedades, y no sólo algunos bancos y empresas, han entrado en bancarrota² ${ }^{2}$.

Según Fernández Buey, el modelo de civilización capitalista, que ha llevado en nuestros días a instituir a nivel planetario una serie de valores, entra en crisis en el momento en que se constata el profundo deterioro ecológico que provoca. Este deterioro está vinculado con la depredación de recursos naturales a una velocidad incompatible con la requerida por la naturaleza para poder regenerarlos. Un caso paradigmático, aunque no el único, es el de los combustibles fósiles, de los que depende de manera inexorable un modelo de producción, distribución y consumo como el actual, sumamente energívoro y en continuo crecimiento.

Además de la crisis ecológica, la ecofeminista Yayo Herrero denuncia otras dos crisis que ponen de manifiesto el agotamiento del presente modelo civilizatorio, a saber: la crisis social y la crisis de cuidados. Sobre la "crisis social" señala lo siguiente:

El sistema económico basado en el crecimiento continuado se ha mostrado incapaz de satisfacer las necesidades vitales de la mayoría de la población. Hasta el presente los sectores sociales con más poder y más favorecidos han podido superar los límites de sus propios territorios recurriendo a la importación de biodiversidad y "servicios ambientales" de otras zonas del mundo poco degradadas y con abundancia de recursos. Pero esto está dejando de ser así, y estas áreas también se comienzan a deteriorar, agravando la situación de las poblaciones más empobrecidas del mundo que llevan ya décadas sufriendo esta guerra ambiental encubierta ${ }^{3}$.

El modelo de civilización en el que vivimos, a pesar de esquilmar los recursos naturales de muchos territorios, no es capaz de proveer a la humanidad en su conjunto de los bienes básicos para su supervivencia, poniendo en riesgo muchas vidas humanas. Mientras los sectores más privilegiados sobreconsumen recursos a costa

F. Fernández Buey, “Crisis de civilización”, Papeles, n¹05, 2009, p. 41.

Y. Herrero, "Propuestas ecofeministas para un sistema cargado de deudas", Revista de Economía Crítica, n¹3, 2011, p. 42. 
del espolio del sur global, condenando a comunidades enteras al infraconsumo o a la migración, la desigualdad ocasiona también en el norte global la exclusión de la población más desfavorecida, privándola de bienes básicos.

La creciente extracción de recursos no se traduce en mayor nivel de vida de toda la población porque los objetivos socioeconómicos están subordinados en última instancia a un objetivo principal: la acumulación de capital. Esta centralidad de la acumulación se justifica habitualmente aludiendo a dos presuntos beneficios que le acompañan: el desarrollo tecnológico y el incremento productivo ${ }^{4}$. El problema de esta justificación es que obvia los enormes problemas ecológicos que se puedan derivar del desarrollo o el incremento de algunos sectores productivos y, al mismo tiempo, no cuestiona que estén puestos al servicio de la obtención de beneficio económico.

Los pretendidos avances tecnológicos, al promover por encima de todo la ganancia de corporaciones empresariales, siempre supeditan el objetivo de lograr el bienestar de la población a la lógica de la acumulación. Un claro ejemplo lo constituyen los avances en la industria alimentaria o sanitaria que podrían llevar, idealmente, a abastecer de los alimentos fundamentales a la humanidad o a sanar muchas de las enfermedades. Sabemos que este ideal no se realiza y en la práctica los avances de ambas industrias no están dirigidos a estos objetivos. Es más, al estar primordialmente enfocados al incremento de ganancias, estos avances pueden poner en riesgo la salud cuando ello implica un aumento de beneficios o una reducción de gastos, como sucede con la utilización de determinados plaguicidas en las plantaciones ${ }^{5}$ o en el abandono de la investigación de aquellas enfermedades poco rentables ${ }^{6}$.

Al priorizar la acumulación ilimitada de capital sobre la sostenibilidad ecológica o el bienestar de las poblaciones, el sistema socioeconómico entra en conflicto con la vida y las posibilidades de su reproducción; conflicto que se ha venido llamando desde posturas ecofeministas el conflicto capital-vida ${ }^{7}$. Este sistema socio-económi$\mathrm{co}$, al no tener en cuenta en el constante proceso de crecimiento y acumulación los límites temporales y materiales de lo vivo, se presenta como biocida, estableciendo una amenaza constante sobre la vida.

A juicio de Herrero, el cambio de este paradigma de civilización debe ir acompañado de una aceptación de los límites, de la naturaleza en general y del propio cuerpo humano en particular, asumiendo su finitud y vulnerabilidad.

El cuerpo humano es vulnerable por múltiples razones, entre ellas porque depende de otras vidas (humanas y no humanas) para su mantenimiento. No obstante, el actual sistema socioeconómico erige como valor la autosuficiencia e invisibiliza nuestra interdependencia, provocando lo que Herrero denomina "crisis de cuidados".

Invisibilizar nuestra necesidad de cuidado y apoyo por parte de otras personas no la hace desaparecer por arte de magia sino, más bien, provoca que nuestra interdependencia se resuelva en términos de explotación y desigualdad en ámbitos privados

$4 \quad$ El beneficio del incremento productivo está asumido en aquellos estudios que equiparan el bienestar con el producto interno bruto (PIB). Este indicador presenta como positivo cualquier producción y gasto sin tener en cuenta, de manera negativa, los desastres ecológicos que pueden derivarse de ellos.

5 Cf. A. M. del Puerto Rodríguez, S. Suárez Tamayo, D. E. Palacio Estrada, "Efectos de los plaguicidas sobre el ambiente y la salud", Revista Cubana Higiene y Epidemiología, vol.52, n³, 2014, pp.372-387.

6 Cf. R. G. Zurriaráin, "Investigación biomédica, intereses económicos y divulgación periodística” Revista Latinoamericana de Bioética, 17(2), 2017, pp. 51-62.

7 Cf. A. Pérez Orozco, Subversión feminista de la economía. Aportes para un debate sobre el conflicto capitalvida, Madrid, Traficantes de Sueños, 2014. 
de la luz pública. El modelo de autosuficiencia se apoya en la falta de reconocimiento de una ingente cantidad de trabajos de cuidados que son desigualmente repartidos y están frecuentemente feminizados o racializados. Esto provoca que algunas vidas, las que más se aproximan -siempre de manera ilusoria- al modelo de autosuficiencia, sean más reconocidas, salvaguardadas y cuidadas, incluso en momentos de crisis, mientras que otras vidas se exponen de forma más extrema a la precariedad.

Además de las posibles injusticias que podríamos localizar en este modelo civilizador, uno de los mayores problemas que plantea es que nos impide repensar en común como hacer más habitable nuestra ineludible interdependencia. Solamente cuestionando el modelo de autosuficiencia podremos reconocer y visibilizar la vulnerabilidad de la vida, que si carece de apoyos y cuidados que la sostengan se convierte en inviable e invivible. Este reconocimiento nos permitirá abordar la cuestión de las condiciones de posibilidad irrenunciables para que la vida se mantenga. En definitiva, para hacer frente a la creciente precariedad de nuestro tiempo es necesario pensar nuevas subjetividades, y concepciones de vida digna, que se alejen del ideal precedente.

\section{La búsqueda de alternativas a un modelo socioeconómico en crisis}

Frente a la actual crisis multidimensional (ecológica, de reproducción social y de cuidados), que pone en cuestión el modelo civilizador, necesitamos imaginar y construir en común alternativas que nos doten de un horizonte o utopía que nos sirva para caminar en otra dirección.

Como horizonte alternativo se plantea desde miradas ecofeministas poner en el centro de nuestro sistema socioeconómico la sostenibilidad de la vida y construir en común otra noción de vida (que merezca ser vivida, sostenida y rescatada) que no pase por la explotación o dominación de otras vidas. Para caminar en esta dirección, como hemos visto en el apartado precedente, tendremos que reconocer nuestra ineludible interdependencia y ecodependencia.

Esta interdependencia y ecodependencia de la vida humana ha sido negada en un momento determinado en la época moderna, concretamente en la transformación capitalista, favoreciendo el modelo de desarrollo y el mito del crecimiento económico que ha provocado la actual situación de crisis multidimensional. Por ello, desde algunas perspectivas ecologistas y feministas se ha intentado buscar referentes en modelos a los márgenes del proyecto modernizador capitalista. La intención no consistiría en idealizar esos modelos sino intentar rescatar ciertos aspectos de sus concepciones vitales que nos pueden servir de apoyo para construir un nuevo porvenir, de tal manera que no comencemos a caminar desde una tabula rasa y tengamos otros referentes a la vista.

Entre estos referentes se encuentran las concepciones del Buen Vivir en América Latina.

\section{Concepciones del Buen Vivir en América Latina}

La idea de Buen Vivir según los movimientos indígenas de América Latina es una cosmovisión ancestral de la vida que tiene su origen en los saberes y prácticas tradicionales en Abya Yala. Hace referencia a un vivir en armonía con los seres humanos 
y la naturaleza, entendida ésta no como algo ajeno a lo humano, sino como algo de lo que formamos parte. La perspectiva que adopta el Buen Vivir no es egocéntrica ni antropocéntrica, ya que reconoce nuestra interdependencia (nuestra dependencia con la comunidad o ayllu ${ }^{8}$ ) y nuestra ecodependencia (nuestra dependencia con otras vidas no humanas). La concepción del sujeto desde la concepción del Buen Vivir es, por tanto, relacional ${ }^{9}$.

Esta noción resurge con fuerza en las últimas décadas como una alternativa al sistema capitalista, al mito del desarrollo y del crecimiento económico, jugando un papel importante en movimientos sociales y en iniciativas gubernamentales de varios Estados latinoamericanos. De hecho, la palabra en quechua que se refiere a esta idea, Sumak Kawsay, constituye una de las bases de la constitución de Ecuador del año 2008 y la palabra aimara, Suma Qamaña, de la constitución de Bolivia del año 2009.

\subsection{El Buen Vivir como alternativa al desarrollo}

El modelo de desarrollo se extendió durante la segunda mitad del pasado siglo por todo el orbe. Alberto Acosta, expresidente de la Asamblea Constituyente de Ecuador y economista ecuatoriano, señala a este respecto que

Desde mediados del siglo XX un fantasma recorre el mundo, ese fantasma es el desarrollo. Y a pesar de que la mayoría de personas, con seguridad, no cree en fantasmas, al menos en algún momento ha creído en "el desarrollo", se ha dejado influir por "el desarrollo", ha perseguido "el desarrollo", ha trabajado por "el desarrollo", ha vivido del "desarrollo"[...] y es muy probable que lo siga haciendo en la actualidad ${ }^{10}$.

Acosta localiza la institucionalización de este modelo desarrollista en un momento muy concreto: el 20 de enero de 1949. En esa fecha Harry Truman, el presidente de Estados Unidos, realizó un discurso en el que definía a una parte considerable del mundo como "áreas subdesarrolladas" y defendía la idea de extender en esas regiones los avances científicos y el progreso industrial occidental que, a su juicio, podrían acabar con la miseria e incrementar el nivel de vida de las personas que habitaban esos lugares.

Tras esta aparente declaración de buenas intenciones de Harry Truman se escondía la pretensión de reducir todos los modos de vida a un único modelo socioeconómico, el modelo de desarrollo de las naciones más industrializadas. Esta reducción de los modos de vida se basaba en un paradigma evolutivo que consideraba a las sociedades menos industrializadas como sociedades primitivas que debían ser superadas. En este paradigma evolutivo subyacía una estructura de dominación dicotómica, que establecía jerarquías entre: "desarrollado-subdesarrollado, rico-pobre, avanzado-atrasado, civilizado-salvaje, centro-periferia" "11. Estas dualidades jerár-

Unidad comunitaria compuesta por varias familias entre sí.

Es esta relacionalidad, central en la noción de Buen Vivir, la que provoca que algunos teóricos prefieran hablar de Buen Convivir. Para un análisis filológico del sentido original de la expresión aimara "Suma Qamaña” véase X. Albó, Suma Qamaña = El Buen Convivir, Revista Obets, 4, 2009, pp. 25-40.

10 A. Acosta, Buen vivir: sumak kawsay: una oportunidad para imaginar otros mundos, Quito, Ediciones AbyaYala, 2012, p. 35.

$11 \quad$ Ibidem, p. 39. 
quicas justificaron la posterior asunción de una serie de objetivos y proyectos para alcanzar el ansiado desarrollo, ya que ofrecían una visión reductiva del mundo que obviaba las diferencias culturales y su valor inconmensurable.

En los años que siguieron al discurso de Harry Truman, las bondades del desarrollo fueron asumidas - con ciertas diferencias- por los dos bandos enfrentados en la guerra fría: el bloque capitalista y el denominado socialismo real. El desarrollo, por tanto, se estableció como un lugar común que simplificaba los modos posibles de organización humana a ambos lados del Telón de Acero.

El mundo se dividió entonces entre países desarrollados y países en vías de desarrollo $^{12}$, y cualquier intervención -incluida la militar- de los primeros en los segundos, con la finalidad de favorecer el desarrollo, era considerada justa, incluso motivada por fines humanitarios.

Sin embargo no se hicieron esperar los problemas que deparaba el camino hacia el desarrollo, apareciendo rápidamente un deterioro social y ecológico, tanto a escala local como global. Ante estos inconvenientes no previstos, como señala Acosta,

buscamos alternativas de desarrollo. Le pusimos apellidos al desarrollo (Aníbal Quijano) para diferenciarlo de lo que nos incomodaba, pero seguimos en la senda del desarrollo: desarrollo económico, desarrollo social, desarrollo local, desarrollo global, desarrollo rural, desarrollo sostenible o sustentable, ecodesarrollo, etnodesarrollo, desarrollo a escala humana, desarrollo endógeno, desarrollo con equidad de género, codesarrollo... desarrollo al fin y al cabo ${ }^{13}$.

Estos nuevos modelos de desarrollo, engendrados para tratar de solventar los problemas más inquietantes que surgieron como consecuencia de la adopción de unos objetivos y proyectos supuestamente liberadores, no pusieron en cuestión los pilares fundamentales del desarrollo, entendido como un proceso lineal continuo de crecimiento económico. Por este motivo, Eduardo Gudynas los denomina "desarrollos alternativos"14. A su juicio, estos modelos son insuficientes para resolver los desmanes del desarrollo clásico. Esto sucede porque las críticas al desarrollo, propuestas por estos desarrollos alternativos, terminan por ser fagocitadas por los núcleos conceptuales de la idea tradicional de desarrollo.

Según Gudynas y Acosta, si queremos frenar las consecuencias nefastas del desarrollo, no debemos buscar otros caminos que nos sirvan para llegar al mismo lugar, como hacen las concepciones alternativas del desarrollo, sino poner en cuestión la meta misma que se desea alcanzar. Con el objetivo de llevar a cabo esta crítica, en primer lugar cabría mencionar que el desarrollo, o "mal desarrollo" como lo denomina la ecofeminista Vandana Shiva ${ }^{15}$, consiste en la imposición del modelo occidental a todas las demás culturas, en una estrategia neocolonialista que intenta homogeneizar todas las formas de vida siguiendo los parámetros hegemónicos de occidente. Por otra parte, es necesario denunciar que este ideal desarrollista no es reproducible a

12 La expresión "países en vías de desarrollo", frecuentemente utilizada en el ámbito de la diplomacia, se refiere a esos países que, partiendo de un estado de subdesarrollo (considerado desde este paradigma como un atraso) se encuentran en pleno proceso desarrollo económico, aplicando las políticas necesarias para dicho efecto.

13 Ibidem, p. 41.

14 E. Gudynas, "Debates sobre el desarrollo y sus alternativas en América Latina: Una breve guía heterodoxa" en Más allá del desarrollo, Cali, Ediciones Abya-Yala, 2012, p. 47.

15 Cf. V. Shiva, Abrazar la vida: mujer, ecología y desarrollo, Madrid, Editorial Horas y horas, 1995. 
escala global, desde el momento en que el modo de vida que promueve, depredador de bienes materiales, desatiende los límites inherentes a los ciclos ecológicos.

Si todos los países participaran del estilo de vida occidental, productivista y consumista, no bastarían todos los recursos disponibles en el planeta para colmar todos los deseos y necesidades que promueve. Al no ser universalizable, el estilo de vida propio del paradigma desarrollista no puede ser entendido como un derecho sino como un privilegio, inalcanzable para la mayoría de seres humanos, que no disfrutan de las (supuestas) ventajas del ansiado desarrollo.

Desde el momento que reconocemos que el planeta tiene límites, los pilares sobre los que se mantiene la idea de desarrollo se desmoronan. El crecimiento ilimitado de la producción y de la acumulación de bienes no es posible si dependemos de materiales y energía que son finitos. Esta acumulación, además, suele subordinar cualquier fin a la obtención de beneficios económicos, por lo que resulta ingenuo asumir que este paradigma conducirá a una erradicación del hambre o de la pobreza a nivel planetario, como sostienen todavía hoy los acérrimos defensores del desarro1lo. Frente a estos defensores, los críticos ponen en cuestión que haya merecido la pena la devastación social y ecológica producida por la búsqueda del desarrollo. La meta no era ni deseable ni realizable. Ante el manifiesto fracaso del desarrollo, por tanto, plantean abandonar la vía propuesta por los desarrollos alternativos y buscar alternativas al desarrollo.

Con el fin de emprender un camino que siga la senda marcada por un horizonte postdesarrollista, parten del cuestionamiento de ideas y concepciones de las que se ha nutrido el modelo de desarrollo, que siguen teniendo consecuencias en nuestra manera de conocer el mundo. Un lugar destacado lo ocupa la idea de progreso, que se construyó hace cinco siglos en Europa y sirvió en buena medida para justificar la deriva colonialista. El desarrollo es deudor de la concepción de progreso en la medida en que ambos se conciben como un proceso lineal que establece un estado anterior y posterior.

Poco tiempo después de la aparición de la idea de progreso, con el auge de las teorías mecanicistas, prolifera la idea de que el hombre debe gobernar la naturaleza, entendida como algo que le es ajeno. De este modo se establece una frontera infranqueable entre la vida humana y la naturaleza, que puede ser instrumentalizada y dominada. Estas teorías mecanicistas fueron revolucionarias y resultaban contrarias al saber precedente. Al concebir a la naturaleza como una gran máquina (un inmenso reloj mecánico, compuesto de resortes) susceptible de ser conocida de manera abstracta por medio de la geometría o las matemáticas (saberes que se basan en lo cuantificable) se abandonaba la concepción de la ciencia preexistente, basada en cuestiones cualitativas y el reconocimiento de la complejidad de lo vivo ${ }^{16}$.

A pesar de que en las ciencias contemporáneas el mecanicismo ha sido cuestionado, parece que el imperativo de dominar la naturaleza (concebida como algo externo a la existencia humana) por medio de la técnica y la ciencia, sigue teniendo un gran calado en el imaginario colectivo ${ }^{17}$. Hasta el punto que todavía hoy, a pesar de los

16 A los contemporáneos de Descartes que lo vivo y lo inerte pudieran estudiarse siguiendo el mismo método científico, y atendiendo en exclusiva a las relaciones simples de causa y efecto, no les resultaba en absoluto obvio. La complejidad de lo vivo no parecía subsumible a entes abstractos matemáticos.

17 En la división ontológica entre la naturaleza y lo humano se apoyaron las justificaciones de las lógicas extractivistas que tuvieron lugar en el pasado colonial de América Latina. Lo preocupante según los teóricos ecologistas es que incluso bajo gobiernos de izquierda se sigue justificando en nuestros días el neoextractivismo. 
desastres ecológicos que atestiguamos, sigue imperando la confianza ciega y acrítica en que la ciencia y la técnica pueden resolver cualquier contratiempo en el camino hacia el progreso y el desarrollo.

Como respuesta a estas concepciones del mundo, en nuestros días resurge con fuerza la idea de Buen Vivir como una alternativa al desarrollo, a la idea hegemónica de progreso y a la concepción de la naturaleza heredada del mecanicismo.

El Buen Vivir se vincula a prácticas de resistencia al colonialismo y al mal-desarrollo por parte de las comunidades indígenas de América Latina. Esta vinculación a las prácticas de resistencia desmiente que estas comunidades no hayan sido afectadas por la influencia colonial y desarrollista, por ello tenemos que sospechar de las miradas románticas que se vierten sobre la realidad de estas comunidades. Como señala Acosta:

Lo que interesa aquí es que bajo algunos saberes indígenas no existe una idea análoga a la de desarrollo, lo que lleva a que en muchos casos se rechace esa idea. No existe la concepción de un proceso lineal de la vida que establezca un estado anterior y posterior, a saber, de subdesarrollo y desarrollo; dicotomía por la que deben transitar las personas para la consecución del bienestar, como ocurre en el mundo occidental. Tampoco existen conceptos de riqueza y pobreza determinados por la acumulación y la carencia de bienes materiales ${ }^{18}$.

El Buen Vivir puede ser entendido como un modo de vida comunitario que encuentra discrepancias con el modo de vida promovido por el modelo civilizador occidental. Es más, ese modelo civilizador desde esta perspectiva aparece como colonial y exógeno.

En contraposición a la división ontológica entre lo humano y la naturaleza, propia del modelo civilizador occidental, el Buen Vivir reconoce que la vida humana está en continua relación y complementariedad con otras vidas no humanas. Por ello esta forma de vida cuestiona la concepción atropocéntrica inherente al desarrollo del capitalismo y del socialismo real.

Según los saberes indígenas, el Buen Vivir implica un vivir en armonía con la comunidad y con la naturaleza ${ }^{19}$. De tal forma que las lógicas instrumentalistas, que conciben a la naturaleza como un mero recurso o como un ente al que dominar y explotar, no son asimilables a esta concepción de la vida. Lo mismo sucede con las lógicas de competencia, que rompen con la armonía en la comunidad. El ideal de autosuficiencia, que rige el paradigma de la competencia, no se adecua a una concepción de la vida humana que entiende al "nosotros" como condición de posibilidad del "yo", que es impensable de manera completamente aislada.

A pesar de que existen diversas formas de entender el Buen Vivir, un denominador común de todas ellas consiste en que el Buen Vivir no debería basarse en el mal vivir de otros. El Buen Vivir apunta a una ética de lo suficiente para toda la comunidad, y no solamente para el individuo, por ello no es asimilable a la idea de vivir mejor occidental, que se sostienen gracias a la existencia de explotación de cuerpos y territorios.

\footnotetext{
A. Acosta, op. cit., p. 63.

Ibidem, p. 70 .
} 


\subsection{Reconceptualizaciones del Buen Vivir por parte de los feminismos comunitarios en América Latina.}

La idea de Buen Vivir no pude ser pensada como una "simple invitación para retroceder en el tiempo y reencontrarse con un mundo idílico, inexistente por lo demás" ${ }^{20}$. Esta advertencia de no idealizar las comunidades indígenas originarias podemos encontrarla de manera significativa en algunos feminismos comunitarios de América Latina, como Mujeres Creando Comunidad de Bolivia o las mujeres mayas y xinkas de AMISMAXAJ ${ }^{21}$ de Guatemala.

Julieta Paredes, en su obra Hilando fino desde el feminismo comunitario, pone en cuestión la idea de que el patriarcado en las comunidades indígenas llegó con la colonia $^{22}$. A su juicio para erradicar el patriarcado no basta con volver a la práctica de complementariedad chacha-warmi (hombre-mujer) presente en las comunidades indígenas. Si bien esta práctica tiene su origen en la tradición, no constituye un horizonte al que se desee aspirar, puesto que los roles asociados a las mujeres en esa concepción de complementariedad son considerados de menor valor e importancia. A su juicio, la teoría del chacha-warmi naturaliza la discriminación y opresión de las mujeres al otorgarle mayor respetabilidad y reconocimiento a las tareas realizadas por los hombres, que ostentan privilegios en la comunidad. Frente a este par complementario jerárquico, que en muchas ocasiones entiende el par según el modelo de la pareja heterosexual, Paredes reivindica una complementariedad igualitaria, sin jerarquías, con el fin de alcanzar un equilibrio y armonía en cualquier tipo de comunidad, no solamente la indígena.

A pesar de estas problematizaciones de las prácticas tradicionales en las comunidades, podemos encontrar en el feminismo comunitario una reivindicación de la noción de Buen Vivir como un horizonte transformador, que puede funcionar como una respuesta a la sociedad individualista y desarrollista occidental. Con el fin de reivindicar una noción de Buen Vivir feminista, se señala la necesidad de reconstruir esta noción desde una mirada no androcéntrica. Lorena Cabnal sostiene que

Tanto por los documentos consultados como por los procesos en que he participado, puedo argumentar que mucho del planteamiento, es desde una construcción cosmogónica masculina, además la mayoría de exponentes y pregoneros del movimiento indígena del paradigma en la actualidad "con propiedad y autoridad epistemológica" son hombres ${ }^{23}$.

Esta disimetría en el movimiento indígena pone en evidencia que todavía queda un largo camino para alcanzar la igualdad en las comunidades o ayllus, ya que las mujeres están en desigualdad de condiciones en relación a los hombres. El ayni (re-

\footnotetext{
$20 \quad$ Ibidem, p. 65.

21 Asociación de Mujeres Indígenas de Santa María Xalapán, Jalapa.

22 Esta idea es problematizada también por María Galindo, de Mujeres Creando, en No se puede descolonizar sin despatriarcalizar. En esta obra diferencia dos patriarcados: el occidental o colonial y el precolonial indígena. A su juicio para acabar con el patriarcado en las comunidades indígenas no basta con descolonizarlas, ya que, de ese modo, quizá podría acabarse con el patriarcado occidental pero no con el patriarcado precolonial.

23 L. Cabnal, "Acercamiento a la construcción de la propuesta de pensamiento epistémico de las mujeres indígenas feministas comunitarias de Abya Yala", en Feminismos diversos: el feminismo comunitario, ACSUR-Las Segovias, 2010, pp. 17-18.
} 
ciprocidad entre los miembros de la comunidad), una categoría presente en la idea de Buen Vivir, no está realizada en las comunidades actuales sino que, más bien, ha de ser construida.

\subsection{EI Buen Vivir como horizonte de cambio a escala global}

El proceso de construcción del Buen Vivir parte de una matriz comunitaria de vida situada en una trayectoria de resistencias locales pero al mismo tiempo puede ser una iniciativa de cambio civilizatorio a escala global. Es susceptible de ser trasformador a escala global desde el momento en que realiza una crítica profunda a las teorías vigentes sobre el desarrollo, incluidas las versiones críticas que han ido resucitando a este concepto agregándole apellidos. Como hemos visto, no es una simple alternativa de desarrollo sino una alternativa al desarrollo.

El modelo del Buen Vivir, por otra parte, se diferencia del modelo civilizador hegemónico en que no pretende ser una propuesta indiscutible, un mandato global, $\mathrm{y}$ aunque parte de una visión utópica del futuro andina y amazónica, esta debe "complementarse y ampliarse incorporando otros discursos y otras propuestas provenientes de diversas regiones del planeta, que espiritualmente están emparentadas en su lucha por una transformación civilizatoria" 24 .

Es relevante que la noción de vida no aparezca como sustantivo en el Buen Vivir sino como verbo. En la formulación misma, por tanto, ya está presente una dimensión performativa. El Buen Vivir no hace referencia a una buena vida que sea susceptible de ser constatada en un momento y un lugar concreto, sino que hace hincapié en su dimensión de devenir, lo que permite nuevas reconfiguraciones. El Buen Vivir, por tanto, aparece como una oportunidad para construir colectivamente nuevas formas de vida. Esto nada tiene que ver con la concepción lineal subyacente a la idea de progreso, con un inicio y un fin definidos, ya que estamos ante un paradigma en perpetua construcción y reconstrucción.

\section{El descentramiento de occidente como paradigma civilizador.}

El concepto del Buen Vivir como alternativa al desarrollo es reivindicado por teóricos del Estado español críticos con el modelo civilizador occidental ${ }^{25}$. Este es el caso por ejemplo de la economista Amaia Pérez Orozco que lo utiliza en su obra Subversión feminista de la economía. Al llamar la atención sobre este concepto proveniente de América Latina busca un descentramiento de nuestro punto de vista occidental, que durante demasiado tiempo se ha creído un referente civilizatorio. Sobre como utilizará el concepto de buen vivir, señala lo siguiente

En estas páginas, hablamos de buen vivir para referirnos a una (futura) noción ética y políticamente codificada de vida que merece ser vivida que sea resultado de una discusión radicalmente democrática. Algo que aún no podemos describir porque no hemos tenido ese debate, pero sobre lo que sí podemos ir dando pistas

\footnotetext{
$24 \quad$ Ibidem, p. 61.

25 Estos ejercicios tienen siempre el peligro de realizar una apropiación cultural. No obstante, a mi juicio, es posible evitar una apropiación indebida si los conceptos culturales de referencia no son convertidos en algo trivial.
} 
que sirvan de travesaños. Ha de ser una noción que responda a las condiciones básicas de la existencia de vulnerabilidad, interdependencia y ecodependencia; que tenga una comprensión multidimensional del bien-estar y entienda que este es una experiencia encarnada, pero siempre vivida en colectivo; que respete dos criterios éticos irrenunciables: universalidad y singularidad ${ }^{26}$.

Ante la actual crisis civilizatoria es necesario tomar en consideración otros modelos distintos al hegemónico que nos posibiliten debatir sobre nuevos horizontes transformadores. Es imprescindible cuestionar la jerarquía tradicional entre el centro y la periferia, entre lo avanzado y lo atrasado, entre lo civilizado y lo salvaje y, por supuesto, entre lo desarrollado y lo subdesarrollado. La riqueza de los debates sobre los saberes y prácticas del Buen Vivir, tradicionalmente situados en los márgenes, da cuenta de lo errado de estas dualidades jerárquicas. Las aportaciones de América Latina resultan de gran importancia para repensar en común nuevas formas de vida que tengan en cuenta la relacionalidad de la vida humana con otras vidas (humanas y no humanas). Las expresiones de Sumak Kawsay y Suma Qamaña nos ayudan a pensar en otras formas de vida más allá del paradigma de la autosuficiencia, al poner de manifiesto nuestra ineludible interdependencia y ecodependencia. 
